\begin{tabular}{|c|c|}
\hline Title & Reactivity of solvated electrons in ionic liquid interacting with low-pressure plasmas \\
\hline Author(s) & Inagaki, Y oshinobu; Sasaki, Koichi \\
\hline Citation & $\begin{array}{l}\text { Japanese Journal of A pplied Physics(JJA P), 59(6), } 066001 \\
\text { https://doi.org/10.35848/1347-4065/ab8d4e }\end{array}$ \\
\hline Issue Date & 2020-06-01 \\
\hline Doc URL & http:/hdl.handle.net/2115/81622 \\
\hline Rights & (ㅇ [2020] The Japan Society of A pplied Physics \\
\hline Type & article (author version) \\
\hline File Information & Ionic_liquid_solvated_revised_HUSCAP.pdf \\
\hline
\end{tabular}

Instructions for use 


\title{
Reactivity of solvated electrons in ionic liquid interacting with low-pressure plasmas
}

\author{
Yoshinobu Inagaki* and Koichi Sasaki ${ }^{\dagger}$ \\ Division of Quantum Science and Engineering, Hokkaido University, Sapporo 060-8628, Japan
}

\begin{abstract}
We employed the charge transfer to solvent (CTTS) transition to examine the reactivity of solvated electrons in ionic liquid interacting with plasmas. A dye laser pulse at $225 \mathrm{~nm}$ was injected into N,N,N-trimethyl$\mathrm{N}$-propylammonium bis (trifluoromethylsulfonyl) imide with the addition of potassium iodide, which was irradiated with low-pressure plasmas, and the reaction frequency of solvated electrons produced by the CTTS transition was measured by optical absorption spectroscopy. It was observed that the reaction frequency of solvated electrons was enhanced by the plasma irradiation. The enhanced reaction frequency was more remarkable by the irradiation of a nitrogen plasma than argon and oxygen plasmas. In addition, a higher reaction frequency was observed at a closer distance from the plasma-liquid interface. The UV-vis absorbance spectrum of the ionic liquid was changed by the plasma irradiation, indicating the production of unknown species which had a high reaction frequency with solvated electrons in the ionic liquid.
\end{abstract}

\section{Introduction}

Recently, plasma-liquid interaction attracts much attention because of its potential applications in water treatment, ${ }^{1-3)}$ medicine, ${ }^{4-6)}$ agriculture, ${ }^{7-9)}$ and analysis. ${ }^{10-12)}$ A unique reactive species in plasma-liquid interaction, which is never involved in gas-phase plasma chemistry, is solvated electrons. The importance of solvated electrons is summarized as follows. First, solvated electrons greatly change the chemical reaction paths in liquids. According to the knowledge in radiation chemistry, the standard reduction potential of solvated electron is -2.9 $\mathrm{eV},{ }^{13)}$ and it reacts rapidly with many species having positive reduction potentials. Therefore, the density and the reactivity of solvated electrons link directly with the amount of chemical species generated by the plasma. Second, solvated electrons induced by the plasma irradiation have the potential for industrial applications. For example, solvated electrons work as the reducing agent in the syntheses of various metal nanoparticles from metal ions in liquids. ${ }^{14)}$

The study of solvated electrons has a long history in the field of radiation chemistry. After the first observation of solvated electrons in 1962, ${ }^{15)}$ solvated electrons have been in-

*E-mail: inagaki@eis.hokudai.ac.jp

†Corresponding author, E-mail: sasaki@qe.eng.hokudai.ac.jp 
vestigated by pulse radiolysis ${ }^{15,16)}$ and laser photolysis. ${ }^{17-19)}$ The advantage of these methods is the compatibility with pump-probe techniques. In these methods, solvated electrons are produced instantaneously by the irradiation of pulsed electron or laser beam, and the temporal variation of the solvated electron density is measured by optical absorption spectroscopy. The reaction rate coefficients of hydrated electrons in water with various additives have been determined using these methods. ${ }^{13)}$ On the other hand, there are only limited, uncertain information about energetics, binding motifs, and dynamics of solvated electrons, which are required for unraveling the detailed mechanism of DNA damage. Today, many scientists try to clarify these issues using quantum mechanical calculations ${ }^{20,21)}$ and photoelectron spectroscopy of liquid micro jets. ${ }^{22,23)}$

Although there have been growing interests of solvated electrons in plasma-liquid interaction, the number of experimental investigations on solvated electrons is extremely small. This is due to the difficulty in the detection of solvated electrons in liquids interacting with plasmas. Rumbach and coworkers succeeded in the detection of hydrated electrons by optical absorption spectroscopy using the total reflection geometry at the plasma-water interface..$^{24,25)}$ However, this measurement may need delicate optimization of the experimental system, since a serious noise may be caused by the vibration of the plasma-water interface. According to the probable experimental difficulty, we cannot find further progress in the detection of solvated electrons in liquids interacting with plasmas.

To overcome the problem, in this work, we employed the charge transfer to solvent (CTTS) transition as a method for investigating the kinetics of solvated electrons in liquids interacting with plasmas. The CTTS transition is classified into laser photolysis, where solvated electron are produced by photodetachment of $\mathrm{I}^{-} .^{18,19)}$ Since solvated electrons are produced by the irradiation of pulsed dye laser beam, we can measure the lifetime or the reaction frequency of solvated electrons at any time and any position. In this paper, we report the lifetime of solvated electrons in an ionic liquid interacting with low-pressure argon, oxygen, and nitrogen plasmas. The interaction between an ionic liquid and a low-pressure plasma is utilized in the synthesis of metal nanoparticles. ${ }^{26,27)}$

\section{Experimental methods}

Inductively coupled plasmas (ICP) were produced in a cylindrical vacuum chamber using a one-turn internal antenna which was covered with glass fibers. The diameter of the rf antenna was $12 \mathrm{~cm}$. The antenna was connected to an rf power supply at $13.56 \mathrm{MHz}$ via matching circuit. The vacuum chamber was evacuated below $6.5 \times 10^{-3} \mathrm{~Pa}$ using a turbomolecular pump 
before introducing discharge gases. The gases we used for the plasma production were argon, nitrogen, and oxygen. The discharge gas was introduced into the vacuum chamber using a mass flow controller. The gas pressure ranged between 1.3 and 3.9 Pa. We employed a Langmuir probe for measuring the electron density, the electron temperature, and the ion saturation current of the plasma. The electron density increased with the rf power, while the electron temperatures in argon and nitrogen plasmas were $2-4 \mathrm{eV}$. A quartz cuvette $\left(10 \times 10 \mathrm{~mm}^{2}\right)$ was installed in the vacuum chamber, and it was filled with N,N,N-trimethyl-N-propylammonium bis (trifluoromethylsulfonyl) imide (TMPA-TFSI; KANTO Chemical Co., Inc.). Potassium iodide (KI) was dissolved into TMPA-TFSI at a concentration of 0.5-1 mM. The ionic liquid TMPA-TFSI with KI was irradiated with the plasma for 2-6 min. The electrical potential of the ionic liquid was floating.

The optical arrangement for producing and detecting solvated electrons is schematically shown in Fig. 1. The cuvette with TMPA-TFSI/KI in the vacuum chamber was irradiated with a dye laser pulse at a wavelength of $225 \mathrm{~nm}$ via a quartz window on the vacuum chamber. The duration of the laser pulse was $8 \mathrm{~ns}$. Iodine negative ions $\left(\mathrm{I}^{-}\right)$in the solution were excited to the CTTS band by absorbing laser photons $\left(\mathrm{I}^{-}+h v \rightarrow \mathrm{I}^{*-}\right){ }^{18,19)}$ The excited ions were photodetached, and electrons thus produced were solvated $\left(\mathrm{I}^{*-}+h v \rightarrow \mathrm{I}^{*}+\mathrm{e}_{\text {solv }}\right)$. We mainly used a diode laser at a wavelength of $1080 \mathrm{~nm}$ for measuring the absorbance of solvated electrons. The dye and diode laser beams were overlapped on the same optical axis using a dichroic mirror. The dye laser beam was terminated by the output-side optical window (Pyrex glass), whereas the diode laser beam was detected using a fast InGaAs photodiode with a rise time constant of $2.3 \mathrm{~ns}$. The output from the photodiode was connected to a oscilloscope, and the temporal variation of the transmitted diode laser intensity was recorded. When we measured the absorbance spectrum of solvated electrons, we replaced the diode laser with a continuum Xe lamp. Interference filters with the transmissions at various infrared wavelengths $(800-1300 \mathrm{~nm})$ were placed in front of the photodiode, and the absorbance by TMPS-TFSI was measured as a function of the wavelength.

In addition to the aforementioned pump-probe measurement, we examined UV-vis absorbance spectrum of the TMPA-TFSI/KI solution after the irradiation of the plasma. The diode laser was replaced with a deuterium lamp in UV-vis absorption spectroscopy, and the transmitted lamp light was detected using a small spectrograph (Ocean Optics, USB2000+). We obtained the absorbance spectrum of the solution by comparing the spectra of the incident and transmitted lamp lights. 


\section{Results}

\subsection{Absorbance waveform and spectrum}

Typical temporal variations of the absorbance, which is defined by $-\log _{10}\left(I_{\mathrm{t}} / I_{0}\right)$ with $I_{0}$ and $I_{\mathrm{t}}$ being the intensities of incident and transmitted diode laser beam, respectively, are shown in Fig. 2. The absorbance increased rapidly in the duration of the dye laser pulse, and after that, we observed the exponential decay as shown in the figure. The absorbance at the peak in the temporal variation is plotted in Fig. 3 as a function of the wavelength. Since the broadband spectrum agrees with the absorbance spectrum of solvated electrons in TMPA-TFSI ${ }^{18,{ }^{19)}}$ it is confirmed by Fig. 3 that the CTTS transition in the TMPA-TFSI/KI solution worked well and we really detected solvated electrons.

The absorbance is proportional to the concentration of solvated electrons according to the Beer's law. Hence, we can deduce the reaction frequency or the reciprocal of the lifetime of solvated electrons by fitting the decay curve shown in Fig. 2 with an exponential function. The reaction frequency of solvated electrons was $5.8 \mathrm{MHz}$ when TMPA-TFSI/KI was not irradiated with the plasma. This reaction frequency coincides well with the value reported in literature. ${ }^{18,19)}$ On the other hand, when TMPA-TFSI/KI was irradiated with an argon plasma which was produced at an rf power of $110 \mathrm{~W}$ and a pressure of $1.3 \mathrm{~Pa}$, as shown in Fig. 2, we observed a higher reaction frequency of $14 \mathrm{MHz}$ for solvated electrons.

\subsection{Increase in reaction frequency by irradiation of argon plasma}

We produced argon plasmas at various rf powers between 20 and $110 \mathrm{~W}$, and examined the reaction frequency of solvated electrons as a function of the electron density. The pressure of argon was fixed at 1.3 Pa. Figure 4 shows the reaction frequencies of solvated electrons observed at various distances from the plasma-liquid interface. As shown in the figure, the reaction frequency increased almost linearly with the electron density. In addition, we observed a higher reaction frequency at a closer distance from the plasma-liquid interface. The reaction frequencies plotted in Fig. 4 were observed at 2 min after the initiation of the discharge.

Figure 5 shows the temporal variation of the reaction frequency of solvated electrons. In this experiment, we initiated the discharge with a duration of 6 min at $t=0$. The $\mathrm{rf}$ power and the argon pressure were $60 \mathrm{~W}$ and $1.3 \mathrm{~Pa}$, respectively, and the electron density of the plasma was $3.2 \times 10^{10} \mathrm{~cm}^{-3}$. At 6 min after the initiation of the discharge, we terminated the rf power. As shown in the figure, the reaction frequency increased almost linearly with time for $6 \mathrm{~min}$. The reaction frequency of solvated electrons decayed slowly after the termination of the discharge. We observed the reaction frequency which is the same as that of virgin 
TMPA-TFSI/KI at $90 \mathrm{~min}$ after the termination of the plasma.

\subsection{Increase in reaction frequency by irradiations of nitrogen and oxygen plasmas}

Figure 6 shows the reaction frequencies of solvated electrons when TMPA-TFSI/KI was irradiated with nitrogen and oxygen plasmas. The result obtained by the irradiation of the argon plasma is also plotted for comparison. The comparison between argon and nitrogen plasmas is given as a function of the electron density (Fig. 6(a)), while the comparison between argon and oxygen plasmas is given as a function of the ion saturation current (Fig. 6(b)), since the evaluation of the electron density from the voltage-current curve of the Langmuir probe was difficult in the oxygen plasma. Higher rf powers were necessary to produce the nitrogen and oxygen plasmas with similar electron densities to the argon plasma. As shown in Fig. 6(a), we observed higher reaction frequency of solvated electrons when TMPA-TFSI/KI was irradiated with nitrogen plasma. In addition, a nitrogen plasma produced at a higher pressure resulted in a higher reaction frequency. On the other hand, as shown in Fig. 6(b), the reaction frequency was almost similar when TMPA-TFSI/KI was irradiated with argon and oxygen plasmas.

\subsection{UV-vis absorbance spectrum of TMPA-TFSI/KI solution}

Two types of UV-vis absorbance spectra of the TMPA-TFSI/KI solution, which was irradiated with the argon plasma for 2 min, are shown in Fig. 7(a). The rf power and the argon pressure were $100 \mathrm{~W}$ and $1.3 \mathrm{~Pa}$, respectively, and the electron density of the plasma was $5.3 \times 10^{10}$ $\mathrm{cm}^{-3}$. The blue (ii) and green (iii) spectra were observed immediately and at $60 \mathrm{~min}$ after the termination of the plasma, respectively. The absorbance spectrum of the virgin TMPATFSI/KI solution ((i), the red spectrum) is also shown in Fig. 7(a) for comparison. The peak at $200 \mathrm{~nm}$ is assigned to TMPA-TFSI since the same spectrum is observed in TMPA-TFSI without the addition of KI. The peak at $225 \mathrm{~nm}$ is due to the CTTS transition of $\mathrm{I}^{-}$. As shown in the figure, the irradiation of the plasma made the solution less transparent, and the absorbance spectrum did not recover to the original one even at $60 \mathrm{~min}$ after the termination of the plasma. Figure 7(b) shows the difference in three spectra shown in Fig. 7(a). The red curve shows the difference between spectra (i) and (ii), suggesting the absorbance spectrum of species produced by the irradiation of the argon plasma. We observed the appearance of four peaks at 210, 230, 280, and $360 \mathrm{~nm}$. On the other hand, the blue curve shows the difference between spectra (ii) and (iii), suggesting the absorbance spectrum of species disappeared in the period of $60 \mathrm{~min}$ after the termination of the plasma. We observed the disappearance of 
three peaks at 230,280 , and $360 \mathrm{~nm}$.

\section{Discussion}

\subsection{Absolute density of solvated electrons}

The absolute density of solvated electrons produced by the CTTS transition is readily obtained from the Beer's law using the knowledge on the absorption coefficient. According to the absorption coefficient of $1.9 \times 10^{4} \mathrm{M}^{-1} \mathrm{~cm}^{-1}$ at $1100 \mathrm{~nm},{ }^{18)}$ the concentration of solvated electrons corresponding to the absorbance of 0.1 is $5.2 \mu \mathrm{M}$. Therefore, the concentration of solvated electrons is expected to be much lower than the concentrations of other chemicals in the TMPA-TFSI/KI solution. In other words, it is expected that the chemistry in TMPA-TFSI is not perturbed significantly by the production of solvated electrons. The negligible concentration of solvated electrons is supported by the exponential decay or the first-order reaction kinetics which is realized when the scavenger has a much higher density than solvated electron.

\subsection{Effect of plasma irradiation}

The enhancement of the reaction frequency of solvated electrons was observed by the irradiation of the noble argon plasma as shown in Figs. 4 and 5. In this case, it is supposed that chemicals produced from TMPA-TFSI or KI react with solvated electrons. The argon plasma provides metastable states $\left(\mathrm{Ar}^{\mathrm{M}}\right)$ and positive ions $\left(\mathrm{Ar}^{+}\right)$. The potential energies of the metastable states $\left(4 s[3 / 2]_{2}^{o}\right.$ and $\left.4 s^{\prime}[1 / 2]_{0}^{0}\right)$ are approximately $12 \mathrm{eV}$. The energy corresponding to the ionization potential of argon $(15.8 \mathrm{eV})$ is released from $\mathrm{Ar}^{+}$when it arrives at the plasma-liquid interface. In addition, $\mathrm{Ar}^{+}$has a kinetic energy $(\sim 10 \mathrm{eV})$ since it is accelerated in the sheath electric field. The deposition of these energies may induce dissociation of TMPA-TFSI and/or KI at the plasma-liquid interface, resulting in the production of chemicals which react with solvated electrons. The importance of the productions at the plasma-liquid interface is seen in Fig. 4, where we observed a higher reaction frequency at a closer distance from the plasma-liquid interface. In the comparison between $\mathrm{Ar}^{\mathrm{M}}$ and $\mathrm{Ar}^{+}, \mathrm{Ar}^{+}$is more suitable to explain the linear relationship between the reaction frequency and the electron density (Fig. 4). We expect the saturation of the $\mathrm{Ar}^{\mathrm{M}}$ density with the electron density in the present experimental condition, considering the kinetics of collisional quenching by electrons. ${ }^{28)}$

The contribution of reactive neutral species to the enhancement of the reaction frequency was clearly observed by the irradiation of the nitrogen plasma as shown in Fig. 6(a). The reactive species provided from the nitrogen plasma is atomic nitrogen, molecular nitrogen at the metastable $A^{3} \Sigma_{u}^{+}$state, and molecular nitrogen ion. According to our previous work, both the 
densities of atomic nitrogen and molecular nitrogen at the metastable state increased almost linearly with the rf power in nitrogen plasmas which were produced at similar conditions to the present work. ${ }^{29)}$ Hence, we cannot judge the most important reactive nitrogen species on the basis of the present experimental data. On the other hand, according to Fig. 6(b), reactive oxygen species are less important than reactive nitrogen species. The selective reaction may be due to the complicated molecular structure of TMPA-TFSI. Another possibility to explain the difference in the reaction frequencies in the plasmas with the different gases is the destruction of solvated electrons by the irradiation of photons from the plasmas, since the argon, nitrogen, and oxygen plasmas have different optical emission spectra. The destruction of solvated electrons is observed in TMPA-TFSI by the irradiation of a Nd:YAG laser pulse at $532 \mathrm{~nm} .{ }^{30)}$ However, this destruction process is unlikely in the present experimental condition, since the absolute photon flux from the plasmas is several orders of magnitude smaller than that of the pulsed Nd:YAG laser. ${ }^{31)}$

\subsection{Scavenger produced in TMPA-TFSI/KI solution}

It is difficult to identify the scavenger chemical that contributes to the enhancement of the reaction frequency of solvated electrons on the basis of the experimental data obtained in the present work. It is understood from Fig. 5 that the scavenger chemical is not stable species. The lifetime of the scavenger chemical is estimated to be $15 \mathrm{~min}$ from the decay curve of the reaction frequency. The chemicals produced by the irradiation of the argon plasma had absorbance peaks at 210, 230, 280, and $360 \mathrm{~nm}$, as shown in Fig. 7(b), but the chemical having the absorbance at $210 \mathrm{~nm}$ does not contribute to the reaction frequency of solvated electrons since it still exists at $60 \mathrm{~min}$ after the termination of the plasma. By comparing Fig. 7(b) with the spectrum reported in literature, ${ }^{32)}$ the peaks at 280 and $360 \mathrm{~nm}$ are assigned to $\mathrm{I}_{3}^{-}$. A production process of $\mathrm{I}_{3}^{-}$originates from the CTTS reaction, namely, atomic iodine produced by $\mathrm{I}^{-}+h v \rightarrow \mathrm{I}+\mathrm{e}_{\text {solv }}$ reacts with $\mathrm{I}^{-}$to form $\mathrm{I}_{2}^{-}\left(\mathrm{I}+\mathrm{I}^{-} \rightarrow \mathrm{I}_{2}^{-}\right)$and it is followed by $\mathrm{I}_{2}^{-}+\mathrm{I}_{2}^{-} \rightarrow$ $\mathrm{I}_{3}^{-}+\mathrm{I}^{-} \cdot{ }^{33)}$ Another production mechanism of $\mathrm{I}_{3}^{-}$is the plasma irradiation. The production of $\mathrm{I}_{3}^{-}$is observed in a KI-starch solution irradiated with an atmospheric-pressure plasma jet. ${ }^{34)}$ We examined the reaction frequency of solvated electrons in the TMPA-TFSI/KI solution with various concentrations of $\mathrm{I}_{3}^{-}$. The concentration of $\mathrm{I}_{3}^{-}$was controlled by adding various amounts of $\mathrm{I}_{2}$ into TMPA-TFSI/KI, where $\mathrm{I}_{3}^{-}$is produced by $\mathrm{I}^{-}+\mathrm{I}_{2} \rightarrow \mathrm{I}_{3}^{-}$. As a result, we found that the reaction frequency of solvated electrons was independent of the concentration of $\mathrm{I}_{3}^{-}$. Therefore, we can conclude that the chemical which has the absorbance at $230 \mathrm{~nm}$ is the scavenger of solvated electrons. We checked literature, but unfortunately we have not 
identified the scavenger chemical yet.

\section{Conclusions}

In this work, we employed the CTTS transition to investigate the reaction frequency of solvated electrons in an ionic liquid interacting with low-pressure plasmas. We observed the increase in the reaction frequency by the plasma irradiation. The increase was roughly proportional to the electron density of the plasma. The irradiation of the nitrogen plasma enhanced the reaction frequency more remarkably than the argon and oxygen plasmas. In addition, a higher reaction frequency was observed at a closer distance from the plasma-liquid interface, suggesting the production of the scavenger chemical at the plasma-liquid interface by the irradiation of ionic and neutral species from the plasma. We have already shown that the experimental technique reported in this paper is applicable to water interacting with an atmospheric-pressure plasma, and the experimental results of hydrated electrons will be reported in a separate paper.

\section{Acknowledgment}

This work was supported by JSPS KAKENHI Grant Numbers 19K21861 and 16H02121. 


\section{References}

1) T. Miichi, N. Hayashi, S. Ihara, S. Satoh, and C. Yamabe, Ozone Sci. Eng. 24, 471 (2002).

2) Z. Stará, and F. Krčma, Czech. J. Phys. 54, C1050 (2004).

3) B. R. Locke, M. Sato, P. Sunka, M. R. Hoffmann, and J.-S. Chang, Ind. Eng. Chem. Res. 45, 882 (2006).

4) G. Fridman, G. Friedman, A. Gutsol, A. B. Shekhter, V. N. Vasilets, and A. Fridman, Plasma Process. Polym. 5, 503 (2008).

5) M. G. Kong, G. Kroesen, G. Morll, T. Nosenko, T. Shimizu, J. van Dijk, and J. L. Zimmermann, New J. Phys. 11, 115012 (2009).

6) K. -D. Weltmann, M. Polak, K. Masur, T. von Woedtke, J. Winter, and S. Reuter, Contrib. Plasma Phys. 52, 644 (2012).

7) S. Perni, D. W. Liu, G. Shama, and M. G. Kong, J. Food Prot. 71, 302 (2008).

8) B. Šerá, I. Gajdová, M. Šerý, and P. Špatenka, Plasma Sci. Technol. 15, 935 (2013).

9) R. Thirumdasa, A. Kothakotab, U. Annapurec, K. Siliverud, R. Blundelle, R. Gattf, and V. P. Valdramidisg, Trends Food Sci. Technol. 77 , 21 (2018).

10) N. Shirai, S. Uchida, and F. Tochikubo, Plasma Sources Sci. Technol. 23, 054010 (2014).

11) P. Rumbach, A. E. Lindsay, and D. B. Go, Plasma Sources Sci. Technol. 28, 105014 (2019).

12) Y. E. Kovach, M. C. Garca, and J. E. Foster, IEEE Transaction Plasma Sci. 47, 3214 (2019).

13) G. V. Buxton, C. L. Greenstock, W. P. Helman, and A. B. Ross, J. Phys. Chem. Ref. Data 17, 513 (1988).

14) Q. Chen, J. Li, and Y. Li, J. Phys. D: Appl. Phys. 48, 424005 (2015).

15) E. J. Hart and J. W. Boag, J. Am. Chem. Soc. 84, 4090 (1962).

16) J. F. Wishart and P. Neta, J. Phys. Chem. B 107, 7261 (2003).

17) F. Barat, L. Gilles, B. Hickel, and B. Lesigne, J. Phys. Chem. 76, 302 (1971).

18) R. Katoh, Y. Yoshida, Y. Katsumura, and K. Takahashi, J. Phys. Chem. B 111, 4770 (2007).

19) K. Takahashi, T. Sato, Y. Katsumura, J. Yang, T. Kondoh, Y. Yoshida, R. Katoh, Radiat. Phys. Chem. 77, 1239 (2008).

20) J. M. Herbert and L. D. Jacobson, J. Phys. Chem. A 115, 14470 (2011). 
21) M. P. Coons, Z. Q. You, and J. M. Herbert, J. Am. Chem. Soc. 138, 10879 (2016).

22) A. T. Shreve, T. A. Yen, and D. M. Neumark, Chem. Phys. Lett. 493, 216 (2010).

23) Y. Yamamoto, S. Karashima, S. Adachi, and T. Suzuki, J. Phys. Chem. A 120, 1153 (2016).

24) P. Rumbach, D. M. Bartels, R. M. Sankaran, and D. B. Go, Nat. Commun. 6, 7248 (2015).

25) P. Rumbach, D. M. Bartels, R. M. Sankaran, and D. B. Go, J. Phys. D: Appl. Phys. 48, 424001 (2015).

26) T. Kaneko, K. Baba, T. Harada, and R. Hatakeyama, Plasma Process. Polym. 6, 713 (2009).

27) M. Bretthole, O. Höfft, L. Klarhöfer, S. Mathes, W. MausFriedrichs, S. Z. E. Abedin, S. Krischok, J. Janek, and F. Endres, Phys. Chem. Chem. Phys. 12, 1750 (2010).

28) K. Sasaki and R. Asaoka, Jpn. J. Appl. Phys. 50, 08JB02 (2011).

29) Y. Horikawa, K. Kurihara, and K. Sasaki, Jpn. J. Appl. Phys. 49, 026101 (2010).

30) K. Takahashi, K. Suda, T. Seto, Y. Katsumura, R. Katoh, R. A. Crowell, and J. F. Wishart, Radiat. Phys. Chem. 78, 1129 (2009).

31) K. Zaima, K. Kurihara, and K. Sasaki, Jpn. J. Appl. Phys. 51, 08 HC02 (2012).

32) Y. Nishiyama, M. Terazima, and Y. Kimura, Chem. Phys. Lett. 491, 164 (2019).

33) K. Takahashi, S. Sakai, H. Tezuka, Y. Hiejima Y. Katsumura, and M. Watanabe, J. Phys. Chem. B 111, 4807 (2007).

34) A. Nakajima, G. Uchida, T. Kawasaki, K. Koga, T. Sarinont, T. Amamo, K. Takenaka, M. Shiratani, and Y. Setsuhara, J. Appl. Phys. 118, 043301 (2015). 


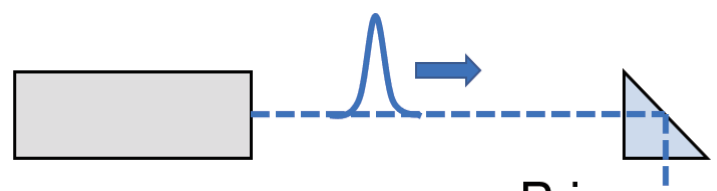

Dye laser pulse 225nm 8 ns
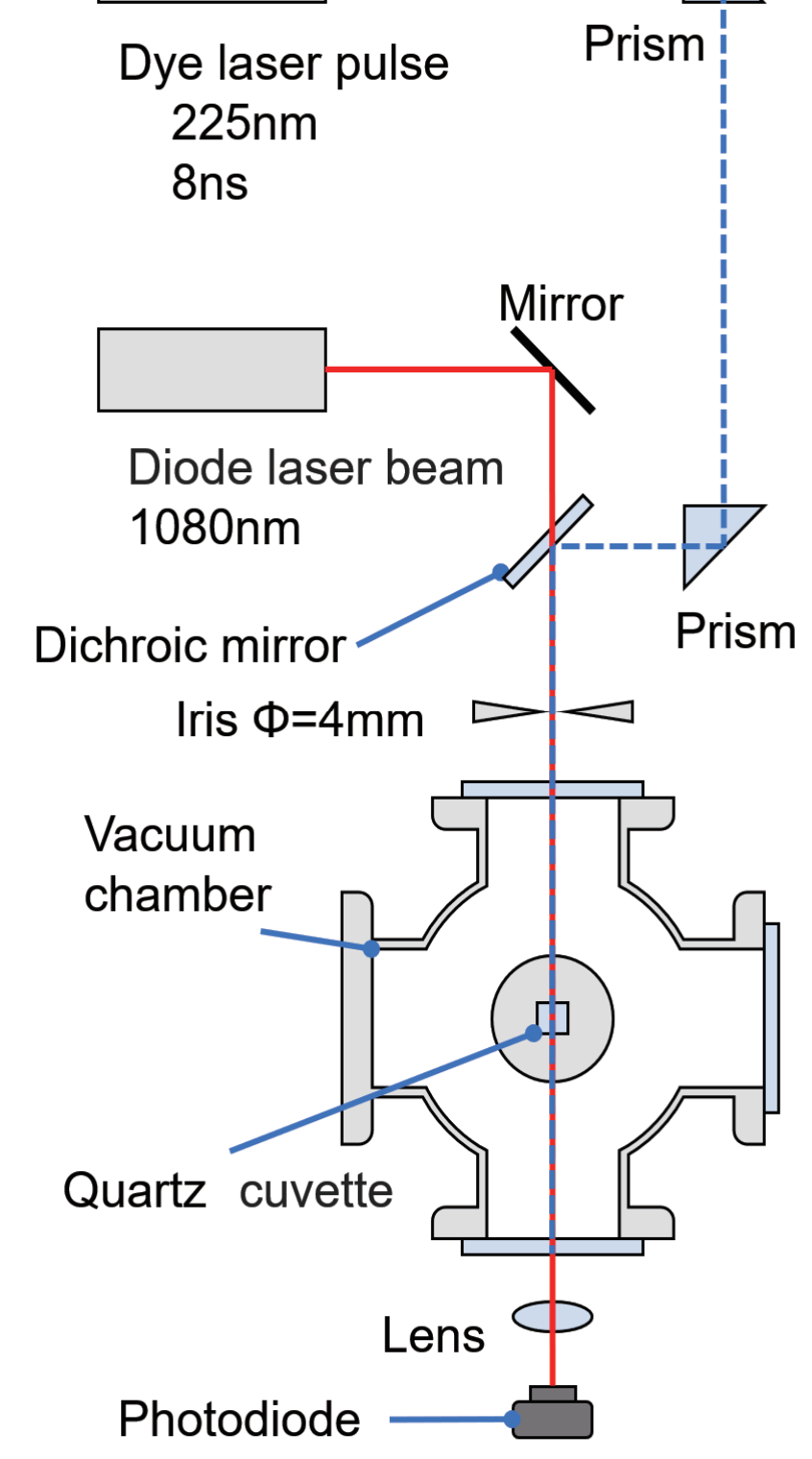

Fig. 1. Optical arrangement for producing and detecting solvated electrons in TMPA-TFSI/KI solution interacting with inductively coupled plasma. 


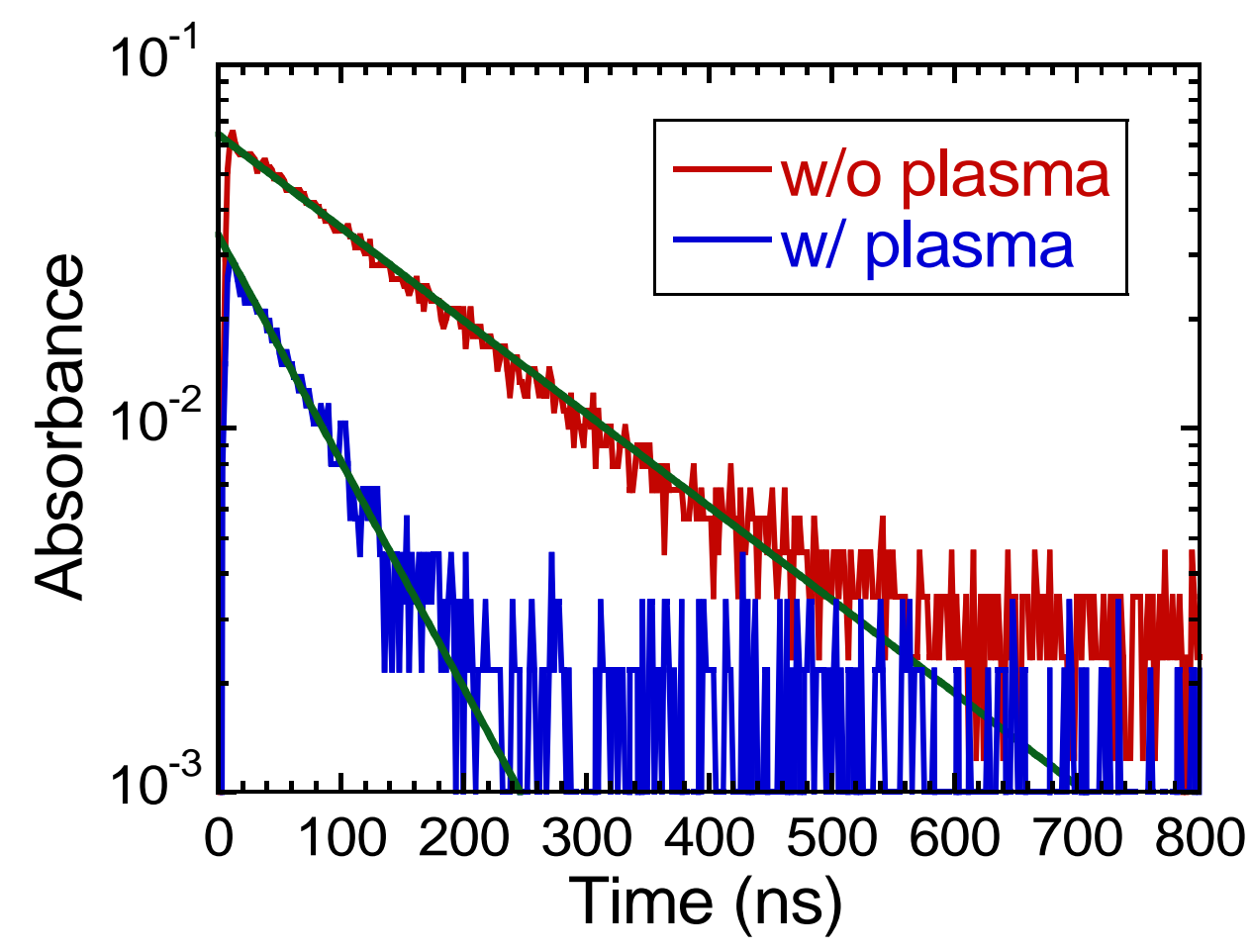

Fig. 2. Temporal variation of absorbance. The dye laser pulse is injected at $t=0$. 


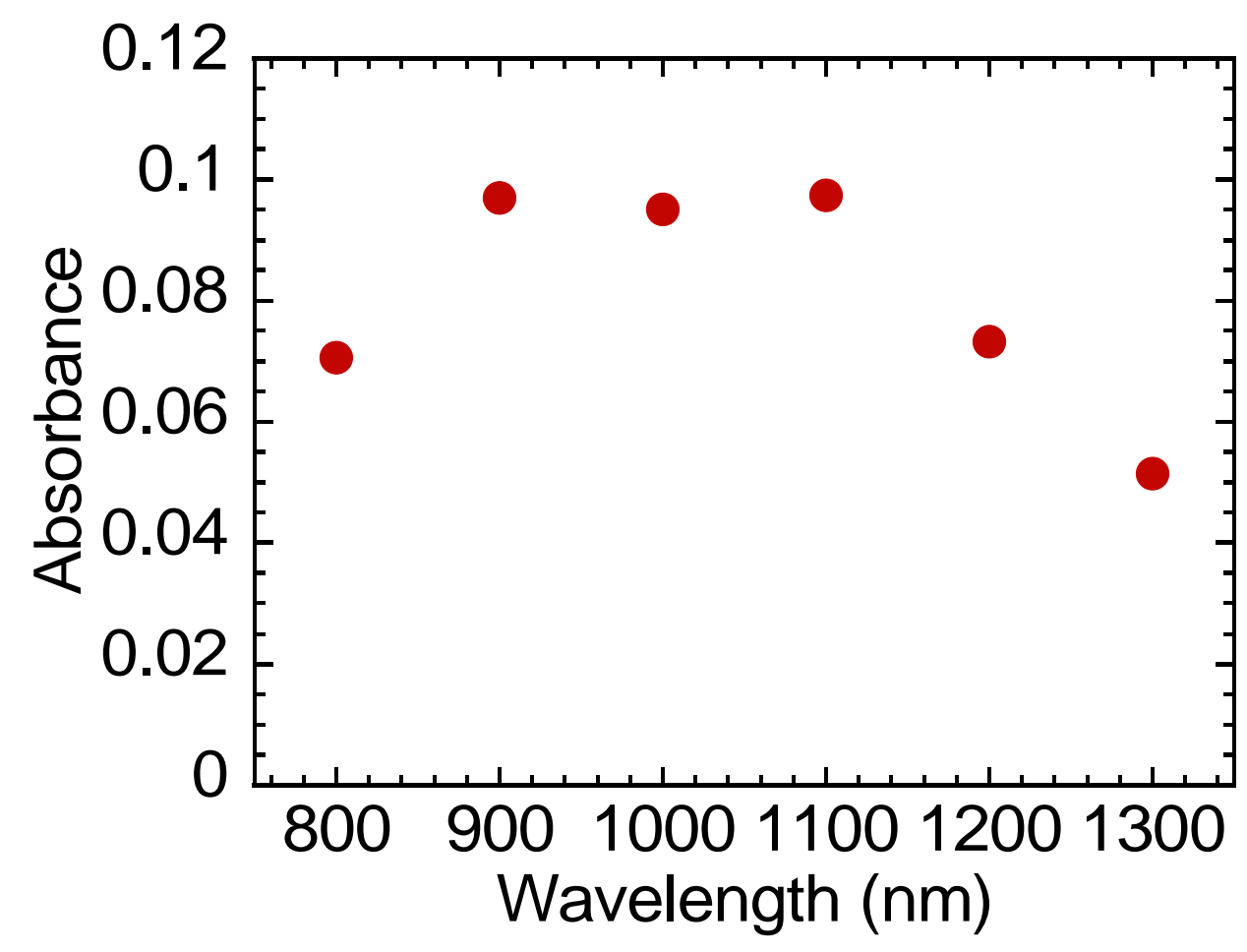

Fig. 3. Spectrum of absorbance. 


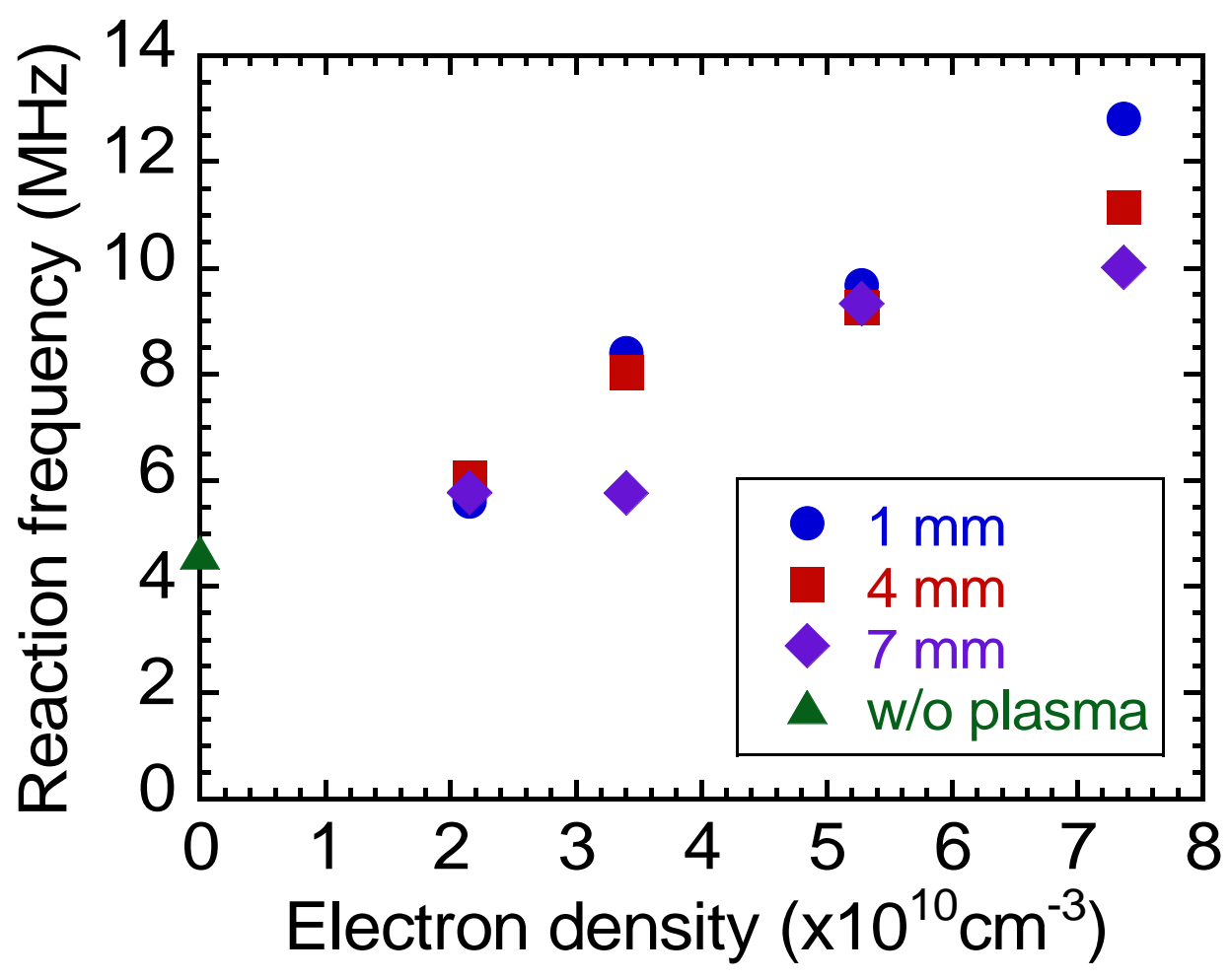

Fig. 4. Reaction frequency of solvated electrons as a function of the electron density of the argon plasma. The measurement was carried out at three distances from the plasma-liquid interface. 


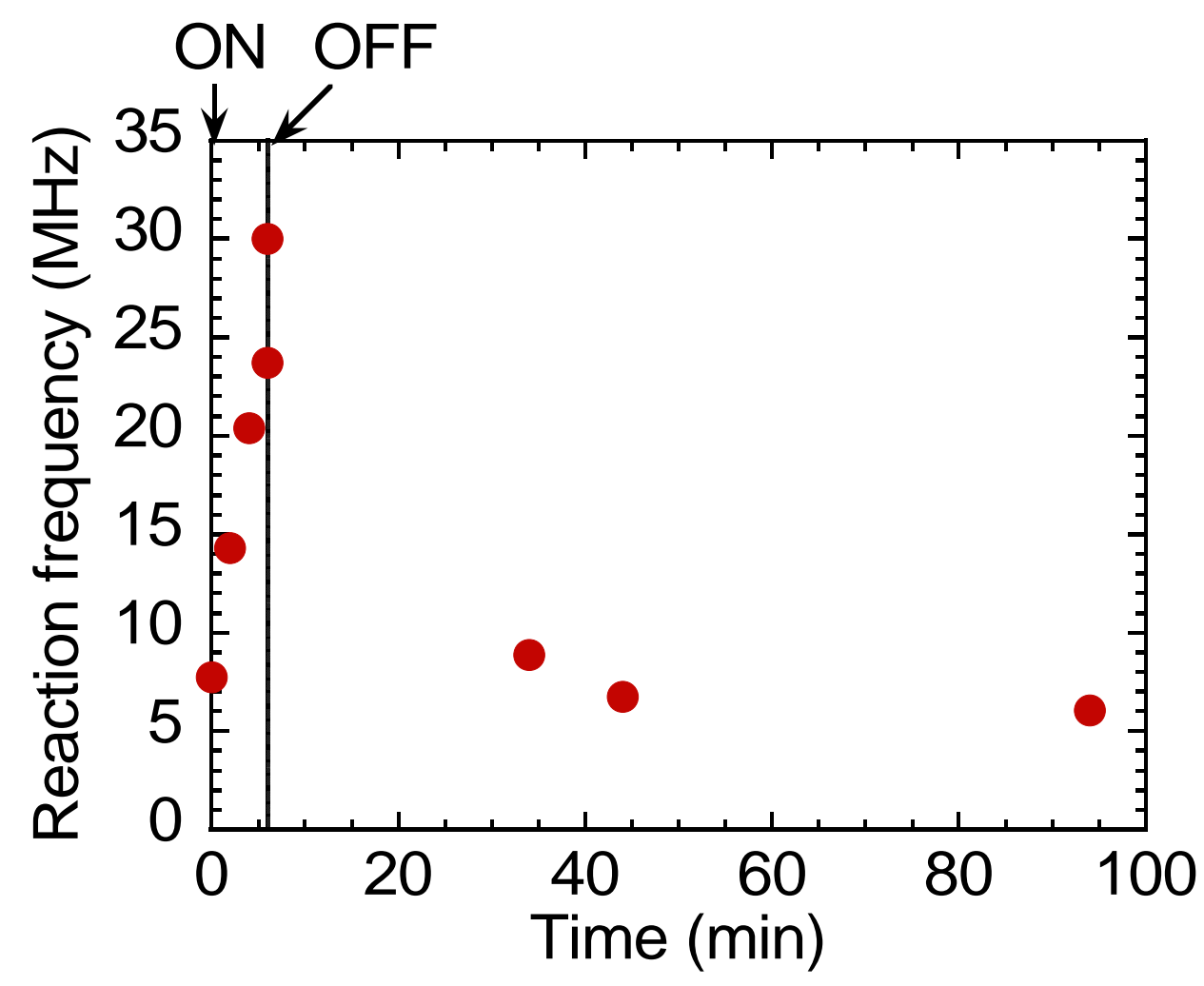

Fig. 5. Temporal variation of the reaction frequency of solvated electrons. The argon plasma was produced between 0 and 6 min. 

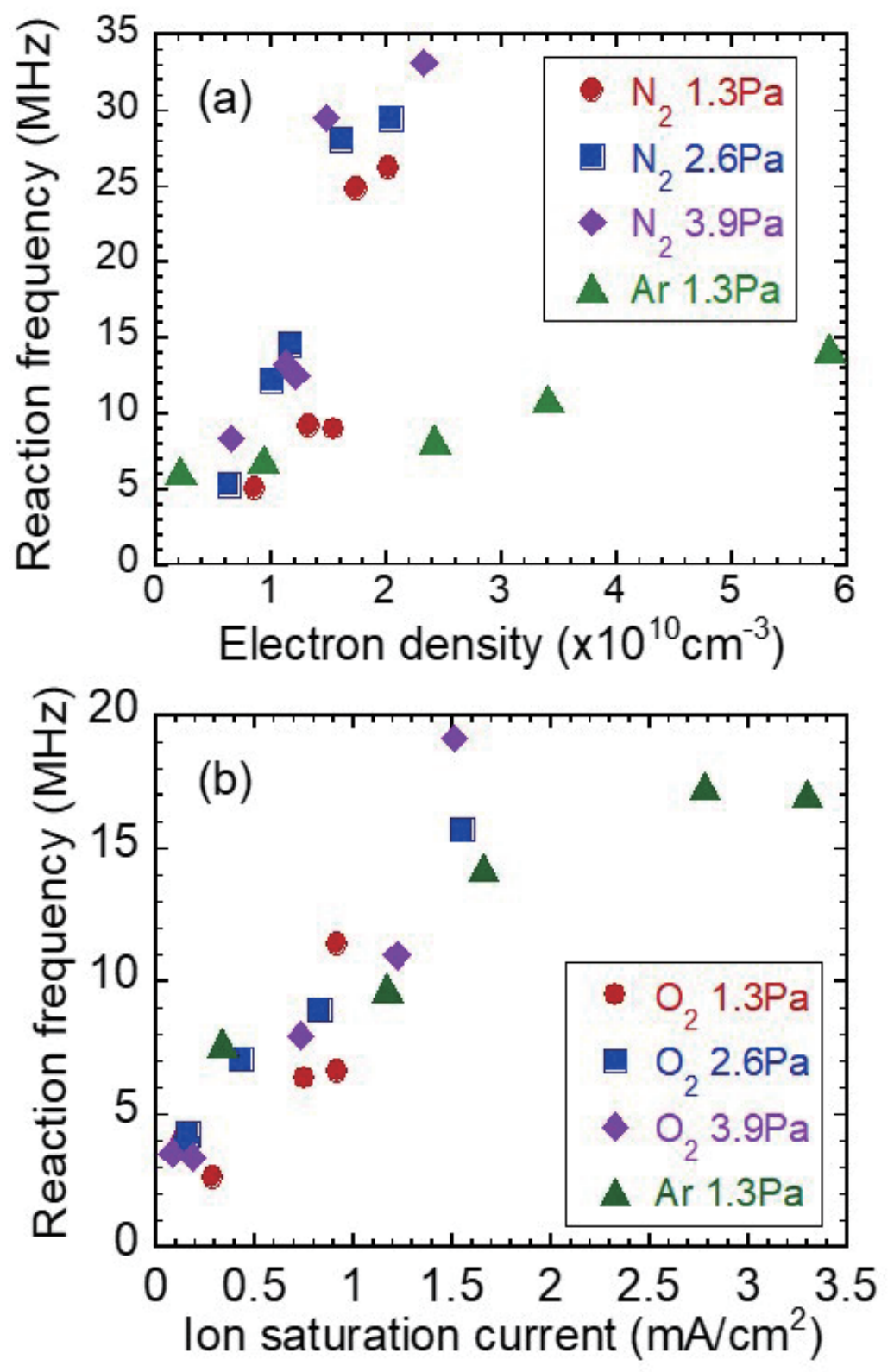

Fig. 6. (a) Reaction frequency of solvated electrons as a function of the electron densities of argon and nitrogen plasmas at various pressures. (b) Reaction frequency of solvated electrons as a function of the ion saturation current of argon and oxygen plasmas at various pressures. 

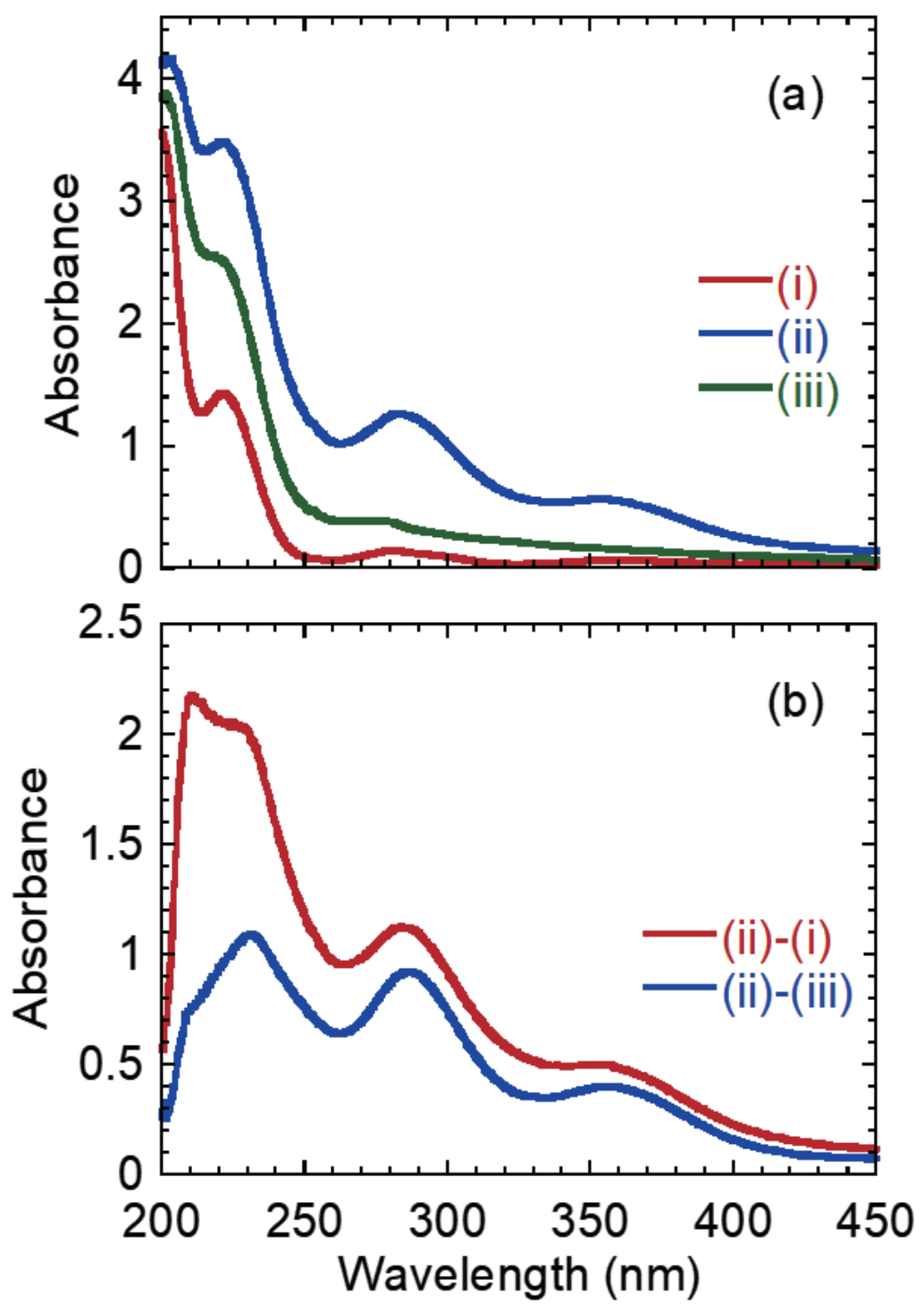

Fig. 7. (a) (ii) and (iii) are absorbance spectra of the TMPA-TFSI/KI solution irradiated with the argon plasma for $2 \mathrm{~min}$. (ii) was observed immediately after the termination of the plasma, and (iii) was observed at $60 \mathrm{~min}$ after the termination of the plasma. (i) shows the absorbance spectrum of the virgin TMPA-TFSI/KI solution. (b) Red curve shows the difference between spectra (i) and (ii). Blue curve shows the difference between spectra (ii) and (iii). 